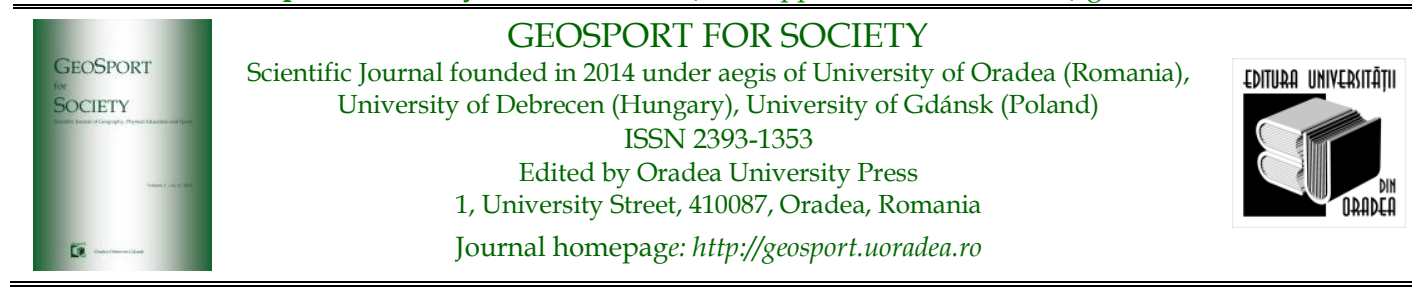

\title{
The Effect of Pilates Stable Device with Instability Device Using the Circuit Training Method on Balance, Flexibility, and Abdominal Muscle Strength
}

\author{
Risfandi SETYAWAN ${ }^{1 *}$, Hari SETIJONO ${ }^{1}$, Nining Widyah KUSNANIK ${ }^{1}$ \\ 1. Sport Science Postgraduate Program Universitas Negeri Surabaya, Indonesia, e-mails: \\ risfandisetyawan@mhs.unesa.ac.id, harisetijono@unesa.ac.id, niningwidyah@unesa.ac.id \\ * Corresponding author \\ Citation: Setyawan, R., Setijono, H., \& Kusnanik, N.W. (2020). The Effect of Pilates Stable Device with Instability \\ Device Using the Circuit Training Method on Balance, Flexibility, and Abdominal Muscle Strength. Geosport for Society, \\ 15(2), 67-81. https://doi.org/10.30892/gss.1501-069
}

Article history: 15.01.2021; Revised: 20.05.2021; Accepted: 20.06.2021, Available online: 14.07.2021

\begin{abstract}
The purpose of this study was to analyze the effect of a stable device with an instability device using circuit training method on balance, flexibility, abdominal muscle strength. This research used quasi-experimental design by giving exercises aided instability devices using a gym ball with a circuit training method in six training posts. Trainer used the gym ball instability devices programmed: 1) bridge on stability ball, 2) single leg on stability ball, 3) hip lift on stability ball, 4) single-leg hip lift on stability ball, 5) stability ball leg rotations stability ball leg rotations 6) prone scaption (Y) on stability ball. Total population is 90 male students, age \pm 19 years, height $\pm 164.40 \mathrm{~cm}$, and body weight $\pm 55.33 \mathrm{~kg}$. From the test of each group (paired sample), it was found that there was a significant difference between pretest and posttest on the variable group of stable devices with instability devices ( $p$-value $>$ $0.05)$, while the control group did not have any difference ( $p$-value $<0.05$ ). Meanwhile, ANOVA test results obtained only the average value of the balance variable in the instability device group using gym ball with control ( $p$-value $0.002<0.05)$, the stable device using a mattress and control ( $p$-value $0.000<0.05$ ), the flexibility group of the stable devices using a control mattress (p-value $0.001<0.05$ ), the abdominal muscle strength device instability using a gym ball with a control ( $p$-value $0.007<0.05<0.05$ ) which has a difference.
\end{abstract}

Keywords: Stable Devices, Instability Devices, Circuit Training, Balance, Flexibility, Abdominal Muscle Strength

\section{Introduction}

The participation of people in sports activities is developed related to healthy lifestyles and achievements. Sports activities also contribute to social development, 
social behavior, fair and sporty play and personal responsibility. Physical activity in multilateral sports requires a strong physical condition component to perform a movement, the ability of the muscles of the body to support one another. Therefore, importance of effective training plan is needed for athletes on proper consideration and supervision. Sports activities also have risk of injury. There is a risk of injury at any age, competitively experiences various impacts of injury to soft tissues, bones, ligaments, tendons, and nerves, which are caused by direct trauma or repeated pressure (Caine and Maffulli, 2005; Maffulli et al., 2010). Physical contact sports, such as rugby, for example, were associated with 5.2 injuries per 1000 of the total athletes involved in high school children. The injuries were more common during competition than exercise, whereas fractures accounted for $16 \%$ of these injuries, whereas concussions (15.8\%) and ligament sprains were almost common (15.7\%) (Collins et al., 2008). The highest injuries were in marathon and sprints. Sports trauma usually affects the joints of the extremities (knees, ankles, hips, shoulders, elbows, wrists) or the spine. Knee injuries are the most common. Knee trauma can cause meniscal and chondral lesions, sometimes combined with cruciatum ligament injury. Ankle injuries constitute $21 \%$ of all sports injuries (Malliaropoulos et al., 2009). Ankle ligament injuries are more common (83\%) diagnosed as ligament sprains, and are common in sports such as basketball and volleyball. Ankle injuries usually occur during competition and in most cases, athletes can do sports within a week (Nelson et al., 2006).

The decreased of balance, skill, coordination and muscle strength have also been found in athletes following lower limb injuries (Zemková and Oddsson, 2015). Balance ability decreased resulting from aging or decreased physical activity can trigger motor skills, which greatly affect mobility in everyday life. Identification of intrinsic risk factors for lower limb muscle injury including previous injury, the influence of age, poor flexibility, and muscle strength decreased or body imbalance (Ekstrand et al., 2011). Every sports movement activities are influenced by the anticipatory ability of the muscles to contract in the torso which is adopted with the demands of the body's instability when doing the movement. Thus, it is very important to maintain physical condition to support functional movement patterns of movement in sports, especially from a perspective related to the ability of balance, strength and flexibility of the body. Other studies have shown that decreased strength of the core muscles of the body can disrupt the balance of the body, thus, instability caused by changes in muscle activation, kinetics and muscle stiffness can have an adverse effect on proprioception and coordination of movements. It was in accordance with (Hrysomallis, 2009), who investigated the relationship between flexibility and injury from hip adductors. The results of this study indicate that flexibility is a parameter affects the risk of injury. Adequate bodily capabilities throughout the body are effective in preventing potential injury.

The right core strengthening exercises for athletes can improve fitness components such as: muscle strength, endurance, agility, speed, balance, and the nervous system, including the vestibular system and the proprioceptive system (Kang, 2015; Yoon et al., 2015) to be more productive and efficient in carrying out movements and less prone to injury. Functionally, the core can be considered very 
important as a kinetic sequence that facilitates the transfer of torque and angular momentum between the lower and upper extremities for specific sport and daily activities in different age groups (Kibler et al., 2006).

Pilates is an exercise program that uses instability and stability devices as a core stability approach to augment the neuromuscular system to control and protect the core body or spine. A method of comprehensively codifying body parts, coordinating core stabilization exercises with challenging mind and breath control with flowing movements of the whole body (Penelope, 2002). Since the Pilates approach focuses on core body exercises and breathe control, it facilitates activation of the transverse abdominis, diaphragm, multifidus and pelvic floor muscles. The joining of these muscles contributes to the stability of the lumbopelvic region. Pilate's exercises have been claimed to be a successful program for core muscle enhancement, rehabilitation. The Research by Harrington and Davies supports that Pilates method improves stem control (Standaert et al., 2008).

The advantages of an unstable training environment will be based on neuromuscular adaptations with increased strength. Increased strength can be associated with an increase in the cross-sectional area of muscles to contract withstand the weight of exercise and to improve neuromuscular coordination (Behm et al., 2011). Another advantage of a gym ball is that it prevents excessive pressure and keeps the spine in a balanced position to avoid low back pain. Therefore, there is consensus on core muscle strengthening or resistance building programs that should involve a destabilizing component. Meanwhile, according to (Shelvam and Singh Sekhon, 2014), recommends the benefits of instability devices using gym balls as a form of exercise that effectively targets core muscles for stability and good posture, but is often overlooked when exercising with simple equipment such as those found in fitness centers (Hyun et al., 2014), in the results of his research that Pilates exercises using a mat were found to be an efficient training method with significant changes in the abdomen and strength, posterior trunk flexibility and abdominal muscle strength in adult women.

Zemková and Oddsson, (2015), a jump that is made on an unstable surface, the level of strength generated in the concentric phase is observed to decrease by $10.3 \%$. (Behm et al., 2002), described a loss of $72 \%$ strength production rate for foot extensors and $21 \%$ for plantar flexxors compared to floor contact and $60 \%$ when performing isometric contractions on the chesh press exercise movement, as well as a decrease in strength. Power and speed of 6-10\% when doing bench press exercises on the surface of the Swiss ball. There is evidence to suggest that exercise on unstable surfaces results in decreased strength, power, and speed of various movements (Behm et al., 2011). Training to increase flexibility, muscle strength, balance and flexibility has been used mostly as an exercise for the Pilates method. In particular, (Sherrington et al., 2008) emphasizes that balance training, lowering the risk of falling by $17 \%$ compared to muscle strength training, balance training should come before other training, an overview of the importance of balance ability.

To improve physical stability, bar stabilization exercises are very helpful for improving balance ability, as the strength of the trunk muscles, among other different factors, is related to balance and functional activity. The activity of the 
trunk muscles maintains balance against gravity, adjusts posture, and prepares for limb movement in daily activities (Verheyden et al., 2006). Most balance exercises are aimed at stabilizing the stem. Pilates workouts also emphasize strengthening the muscles to stabilize the bar through core workouts (Critchley et al., 2011).

Several studies have shown that circuit training are very effective for increasing maximum oxygen consumption, maximum pulmonary ventilation, functional capacity, strength and improving body composition (Brentano et al., 2008). These programs consist of a series, usually 10-15, and resistance training exercises for different parts of the body. For each exercise 12-15 reps, using simple weights (about 40-60\% of one repetition maximum (Romero-Arenas, 2013). Therefore, effect of training on a stable and unstable surface by various training methods needs to be studied in depth. To identify the most effective combination of training forms using a mat and gym ball with the circuit training method on balance, flexibility and strength of the abdominal muscles in core exercise training as a starting point for developing and maintaining a quality physical condition.

\section{Methodology}

The subjects of this research were students of the Physical Education Study of Program of STKIP PGRI Jombang who had received permission from the leadership of the Institute to conduct the research. Each research process was supervised by an instructor, thus bio-ethically this research could be carried out. The research subjects were divided using Ordinal pairing based on pre-test data with the total population of 90 male students, with the characteristics shown in table 1 below;

Table 1. Total population and the characteristics of the research subject

\begin{tabular}{|l|l|l|l|l|}
\hline \multicolumn{2}{|l|}{ Height } & Weight & Body Mass Index & Age \\
\hline Mattress Group & 166,87 & & & \\
\hline Mean & 177 & 58,70 & 21,08 & 18 \\
\hline Max & 156 & 79 & 28,13 & 19 \\
\hline Min & 5,41 & 45 & 15,76 & 17 \\
\hline St. Deviation & 7,42 & 2,53 & 0,83 \\
\hline Gym ball Group & 166,40 & & & 17,87 \\
\hline Mean & 183 & 57,47 & 20,79 & 19 \\
\hline Max & 157 & 79 & 27,66 & 17 \\
\hline Min & 6,37 & 43 & 15,23 & 0,73 \\
\hline St. Deviation & 165,67 & 7,44 & 2,68 & 18,07 \\
\hline Control Group & 175 & 59,73 & 21,74 & 19 \\
\hline Mean & 153 & 95 & 32,87 & 17 \\
\hline Max & 4,76 & 42 & 15,39 & 0,83 \\
\hline Min & & 11,00 & 3,63 & \\
\hline St. Deviation & \multicolumn{2}{|l|}{} \\
\hline
\end{tabular}

This research is quantitative by using a quasi-experimental method. The sample in this study consisted of 3 sample groups, namely the experimental group, which was treated with training on instability devices using a gym ball and stable devices using a mat aided circuit training method and the control group. The total population are 90 male students, with population characteristics aged \pm 19 years, 
height $\pm 164.40 \mathrm{~cm}$, and body weight $\pm 55.33 \mathrm{~kg}$. The research subjects were taken from the population and then divided using ordinal pairing.

This research is an experimental design. The circuit training is done with 6 training posts for the experimental group and one control group. Duration of training is 6 weeks for treatment. Exercise frequency is 3 times per week. Post test measurements were taken 48 hours after the last treatment. While measurement tests: balance beam test, flexibility: Sit and reach test, abdominal muscle strength; partial curl-up.

Program guidelines of stable devices using mats and instability devices using gym ball circuit training methods: total training time \pm 45 minutes, 6 weeks of adaptation duration, training volume $40-60 \%$, number of exercises 6 post circuits, 3 sets number of circuit per session, 3 times per week, of frequency by 30 seconds rest interval, 2 minute break between circuits. The training program develops according to the level difficulty by increasing the reps. Participants take a warm up for 6-8 minutes in every exercise. These stages are supervised by the instructor.

\section{Implementation of Training Programs}

The implementation of training programs with stable device using the mattress have six exercise, which consisted of; (1) bridge on floor, (2) single leg bridging, (3) windshield wiper, (4) unilateral bridge, (5) reverse pendulum, (6) prone scaption $(\mathrm{Y})$ windshield wiper. Meanwhile, the training program with unstable device using gym ball, are; (1) bridge on stability ball, (2) single leg on stability ball, (3) hip lift on stability ball, (4) single-leg hip lift on stability ball, (5) stability ball leg rotations stability ball leg rotations, (6) prone scaption (Y) on stability ball.

\section{Data Analysis}

Data analysis is obtained from the instrument aims to gain conclusions in order to prove whether the stable devices using mattress and instability devices using a gym ball and the circuit training method given has a significant effect on the research subject, using the $\mathrm{Z}$ score, Description of data about the research subject $(\mathrm{N})$, Mean (Mean) of the initial and final tests and the difference between the final test scores and the initial test, paired $\mathrm{T}$ test ( $\mathrm{t}$ test), with the rejection rate of the hypothesis at $\alpha=0.05$. ANOVA (Analysis of Variance), SPSS Statistics 20.

\section{Result}

Table 2. The result of the study

\begin{tabular}{|c|c|c|c|c|c|}
\hline Variable & & & $N$ & Mean & St. Deviation \\
\hline \multirow{6}{*}{ Balance } & \multirow{2}{*}{ Mattress } & Pretest & 30 & 45,10 & 24,23 \\
\hline & & Posttest & 30 & 59,57 & 53,67 \\
\hline & \multirow{2}{*}{ Gym ball } & Pretest & 30 & 41,73 & 33,84 \\
\hline & & Posttest & 30 & 56,10 & 54,45 \\
\hline & \multirow{2}{*}{ Control } & Pretest & 30 & 43,83 & 27,29 \\
\hline & & Posttest & 30 & 40,60 & 36,73 \\
\hline Flexibility & Mattress & Pretest & 30 & 32,42 & 55,68 \\
\hline
\end{tabular}




\begin{tabular}{|c|c|c|c|c|c|}
\hline Variable & \multicolumn{2}{|c|}{ Group } & $N$ & Mean & St. Deviation \\
\hline & & Posttest & 30 & 39,35 & 66,38 \\
\hline & \multirow{2}{*}{ Gym ball } & Pretest & 30 & 31,93 & 63,87 \\
\hline & & Posttest & 30 & 36,93 & 60,99 \\
\hline & \multirow{2}{*}{ Control } & Pretest & 30 & 34,22 & 61,43 \\
\hline & & Posttest & 30 & 37,07 & 62,06 \\
\hline \multirow{6}{*}{ Abdominal Muscle Strength } & \multirow{2}{*}{ Mattress } & Pretest & 30 & 29,37 & 96,15 \\
\hline & & Posttest & 30 & 26,27 & 99,51 \\
\hline & \multirow{2}{*}{ Gym ball } & Pretest & 30 & 28,47 & 70,01 \\
\hline & & Posttest & 30 & 26,30 & 47,28 \\
\hline & \multirow{2}{*}{ Control } & Pretest & 30 & 27,77 & 80,12 \\
\hline & & Posttest & 30 & 26,37 & 76,90 \\
\hline
\end{tabular}

\section{Balance}

In the instability device group using gymbal, it is obtained a mean pretest data of 41.73 seconds with a standard deviation of \pm 33.84 seconds, while the mean posttest data is 56.10 seconds with a standard deviation of \pm 54.45 seconds, in the stable group using a mattress it is obtained a mean The pretest data is 45.10 seconds with a standard deviation of \pm 24.23 seconds, while the mean posttest data is 59.57 seconds with a standard deviation of \pm 53.67 seconds. In control group, the mean pretest data is 43.83 seconds with a standard deviation of \pm 27.29 seconds, while the mean posttest data is 40.60 seconds with a standard deviation of \pm 36.73 .

\section{Flexibility}

In the instability device group using gymbal, the pretest data mean is 31.93 with a standard deviation of 66.38, meanwhile, the mean posttest data is 36.93 with a standard deviation of 60.99, in the stable device group using a mattress it is obtained mean pretest data is 32.42 with a standard deviation of 55.68, meanwhile, the mean posttest data is 39.35 with a standard deviation of 66.38. In control group, the mean pretest data is 34.22 with a standard deviation of 61.43, Meanwhile, the mean posttest data is 37.07 with a standard deviation of 62.06 .

\section{Abdominal Muscle Strength}

In the instability device group using gymbal, the mean pretest data is 28.47 with a standard deviation of 70.01, meanwhile, the mean posttest data is 26.30 with a standard deviation of 47.28, In the stable device group using a mattress the mean data is obtained: pretest is 29.37 with a standard deviation of 96.15 , meanwhile, the mean posttest data is 26.27 with a standard deviation of 99.51. In the control group, the mean pretest data is 27.77 with a standard deviation of 80.12 . Meanwhile, the mean posttest data is 26.37 with a standard deviation of 76.90 .

The Tukey HSD test shows that there is a difference of increasing between the three groups for each variable. This difference can be seen from the mean difference. Based on the mean difference value for the balance variable that the stable device group using the mattress has better improvement than the instability device group using the gym ball with a mean difference of 3.467 and the control group with mean difference of 18.967. For the flexibility variable that stable device group used a 
mattress, the increase was better than the instability device group using a gym ball with a mean difference of 2.413 while the control group with a mean difference of 2.277. For the variable of abdominal muscle strength, the increase in the instability group using gym ball was better than the stable device group using a mattress with a mean difference of 0.33 , while the mean difference for the control group was 0.67 .

Table 3. Multiple Comparisons (Tukey HSD)

\begin{tabular}{|c|c|c|c|c|}
\hline Dependent Variable & (I) Group & (J) Group & Mean Difference (I-J) & Sig. \\
\hline \multirow{6}{*}{ Balance } & \multirow{2}{*}{ Mattress } & Gym ball & 3.467 & 0.959 \\
\hline & & Control & 18.967 & 0.296 \\
\hline & \multirow{2}{*}{ Gym ball } & Mattress & -3.467 & 0.959 \\
\hline & & Control & 15.500 & 0.441 \\
\hline & \multirow{2}{*}{ Control } & Mattress & -18.967 & 0.296 \\
\hline & & Gym ball & -15.500 & 0.441 \\
\hline \multirow{6}{*}{ Flexibility } & \multirow{2}{*}{ Mattress } & Gym ball & 2.413 & 0.306 \\
\hline & & Control & 2.277 & 0.348 \\
\hline & \multirow{2}{*}{ Gym ball } & Mattress & -2.413 & 0.306 \\
\hline & & Control & -0.137 & 0.996 \\
\hline & \multirow{2}{*}{ Control } & Mattress & -2.277 & 0.348 \\
\hline & & Gym ball & 0.137 & 0.996 \\
\hline \multirow{6}{*}{ Muscle Strength } & \multirow{2}{*}{ Mattress } & Gym ball & -0.033 & 1.000 \\
\hline & & Control & -0.100 & 0.999 \\
\hline & \multirow{2}{*}{ Gym ball } & Mattress & 0.033 & 1.000 \\
\hline & & Control & -0.067 & 0.999 \\
\hline & \multirow{2}{*}{ Control } & Mattress & 0.100 & 0.999 \\
\hline & & Gym ball & 0.067 & 0.999 \\
\hline
\end{tabular}

\section{Discussion}

Analysis of stable devices using a mattress with the circuit training method on balance, flexibility and endurance of the abdominal muscles

The concept of stable device exercise using the mattress itself focuses on the core or force that activates the local muscles, especially the transverses abdominals, internal oblique muscles, diaphragm, lumbar multifidus, and pelvic floor muscles. Currently, scientific data shows that these muscles have a major role in stabilizing the lumbo-pelvic system. The stability of the lumbo-pelvic system also depends on the central nervous system to form a stability response with transverse abdominals contractions that have been programmed to stabilize the spine. With a stable device using a mattress the spine is more compressed when the hips are challenged by internal and external forces, as a result of the reactive forces of moving the upper and lower limbs during each exercise position.

Stable device exercises using a mattress focus on maintaining the 'spine in a neutral position', pelvic and spinal stability, by increasing balance and postural stability, these exercises resemble the weight training theory for optimal strength and endurance. This method is comprehensive body-mind conditioning, which coordinates core stabilization exercises with challenging mind and breath control 
with flowing movements from the rest of the body. Since the Pilates approach focuses on core body exercises and breathe control, it facilitates activation of the transverse abdominals, diaphragm, multifidus and pelvic floor muscles. The joining of these muscles contributes to the stability of the lumbopelvic area. The findings of this study require further investigation into the implications of their effectiveness.

Analysis of stable devices using a mattress with the circuit training method to balance, results of the study show evidence to support the initial hypothesis, with a sig. $0.000<0.05$, which means that there is a significant effect on the ability to balance the position of movement in stable device exercises using a mat to change the activation of the multifidus, gluteus maximus, rectus abdominis, and oblique muscles. TrA primarily stabilize muscle of the lumbopelvic area. TrA continues to contract during the movement of the torso and is responsible for the contraction pattern with the pelvic floor.

The training method using a stable device aided mattress can be used as an appropriate training program to increase flexibility, promote mobility control of the trunk and pelvic segments. It can also help in preventing and reducing injuries and dysfunction of the musculoskeletal system., show that the multifidus and transversus abdominals are more involved with changes in the position of the pelvis and trunk when performing pelvic lifts, whereas the rectus abdominal muscles are primarily responsible for pelvic stability in all knee stretching exercises and for controlling extensor torsion. (Cruz-Ferreira et al., 2011; Freeman et al., 2010; Sherrington et al., 2008), conclude that there is strong evidence to support the use of the Pilates training method at least for increased flexibility, dynamic balance and to increase power, muscle resistance, and reducing the risk of injury by $17 \%$.

Stable devices using a mattress with the circuit training method for flexibility analysis shows that during the 6 week training period contributed to the influence of flexibility ability with sig value of $0.000<0.05$. In the form of a steady exercise device using a matrix to be static and dynamic stretching while the subject is doing exercise at each post circuit (Phrompaet et al., 2011; Segal et al., 2004). Pilates training is a combination of static and dynamic stretching exercises that are appropriate and safe to provide increased hip adductor activation, flexor flexibility, and an increased effect on flexibility abilities (Campos de Oliveira et al., 2015). Pilates training led to a significant increase in body balance and functional mobility, as shown by an increase of 1.81 balance points on the Berg Balance Scale (p: 0.0081) and an increase in balance 1.95 seconds in TUG test ( $p<0.001)$. Studies that have used other measures (Tinetti Test and force plate) reported significant results ( $p$ $<0.05)$. Kloubec, (2010) significant improvement on post-test in two different measures of hamstring flexibility. In the sit and reach test, the increase in flexibility occurred from $30.68 \pm 10.14$ centimeters to $33.41 \pm 8.86$ centimeters, where the supine hamstring flexion test also showed a significant increase in left and right hamstring flexibility from $88.41 \pm 13.20$ degrees to $99.09 \pm 8.57$ degrees and $93.14 \pm$ 12.48 degrees to $102.41 \pm 10.45$ degrees, (Sekendiz et al., 2007) also using sit and reach showed a significant increase (from $23.9 \pm 7.5$ centimeters to $31.3 \pm 6.8$ centimeters), after five weeks of Pilates training in adult women. 
The results analysis of stable device training using mattress with the circuit training method on the strength of the abdominal muscles shows the significance value of $0.015<0.05$, which means that there is an influence on the strength of the abdominal muscles. In line with the research results of (Queiroz et al., 2010), shows that multifidus and transverse abdominal contractions are more involved with changes in pelvic and body position during pelvic lifts, whereas the rectus abdominal muscles are responsible for pelvic stability in all knee stretching exercises and for controlling extensor torsion. In line with the findings of (Herrington and Davies, 2005) shows the effectiveness of Pilates in increasing transverse abdominal activation and stabilization process compared to conventional abdominal exercises. Pilates training showed an increase of $83 \%$.

This study indicates that circuit training that focuses on the core muscles must do exercises alternately up or down for a different program sequence using the circuit training method to get the same results regarding the total volume of exercise in each session, perceiving intensity. Similar exercises, regardless of the training frame. These results suggest that progressive fatigue throughout a training session affects muscle performance. These training characteristics make the muscles well adapted to endurance activities over 40 minutes. The size or cross-sectional area of type I muscles increases as a result of an increase in the mitochondrial filaments, membranes and in the muscle fibers.

A physiological enhancement of core muscle control is achieved by modifying the pelvic and trunk postures. These exercises are performed pushing (in the hip extension phase) and repelling (in the hip flexion phase). As described in the data analysis, a significant effect was obtained from the value of training for 6 weeks with a frequency of 3 times per week, a stable device using a mattress aided the circuit training method in this study causing a physiological effect. With an average training duration of 45 minutes with low to moderate intensity, it is devoted to improving balance, strength, flexibility and muscle endurance. The application of circuit training in this study uses each of the 6 movement posts, from simple movements to movements with complex complexity. Some researchers have shown that circuitbased training is very effective at increasing maximum oxygen consumption, maximum pulmonary ventilation, functional capacity, and strength, increasing body composition, efficient use of time can lead to proven improvements in physical health and fitness (Brentano et al., 2008; Romero-Arenas, 2013). The study results show that progressive fatigue throughout training sessions with the circuit training method on endurance ability affects muscle performance. (Kloubec, 2010), shows a significant rate of improvement ( $\mathrm{p} \leq 0.05)$, means that Pilates exercise for 12 weeks, for two 60 minute sessions per week significantly increases abdominal endurance, flexibility and upper body muscle endurance.

Stable device using this mattress result is proven to assist the functional stabilization of the spine. In other words, strengthening the transverse abdominal and multifidus muscles, and also increases muscle thickness and improves lumbar stabilization. This increased recruitment and synchronous stimulation of motor units also explains the increase in muscle strength. In addition, the exercises used in this training program can help to avoid the occurrence of excessive loads on the 
bone tissue, protecting the lumbar area from the impact of injury due to movement in training. In a stable device using a mattress using the circuit training method, there was a significant increase in balance, flexibility, and abdominal muscle strength, which was indicated by the result of an increase in data analysis. (Hyun et al., 2014), efficient Pilates training method with significant changes in the improvement of abdominal muscle strength, posterior trunk flexibility and abdominal muscle strength.

Stable devices using a mattress with the circuit training method can be used as a reference of exercise program to improve balance, flexibility, abdominal muscle strength, additional training programs to increase flexibility, improve controlmobility of the trunk and pelvic segments. It is also able to help in preventing and reducing injuries. A coach can consider core stability as the foundation from which his athlete can generate strength. Core stability, in terms of the musculoskeletal system, may be precisely defined as the endurance capacity of a group of core muscles, which work in harmony to stabilize the trunk and provide a framework for good posture and functional movement.

\section{Analysis of instability devices using a gym ball with the circuit training method for balance, flexibility and endurance of the abdominal muscles}

Significant effect of the training value for 6 weeks with a frequency of 3 times per week, the instability device using the gym ball aided circuit training method using 6 forms of exercise by using the gymball. With duration of about 45 minutes with low to moderate intensity specifically in this study, it causes physiological effects of increasing balance, flexibility, back muscle strength, leg muscle strength, abdominal muscle strength, abdominal muscle endurance and leg muscle endurance. The main purpose of training with instability devices is to reduce the area of contact with the ground by forming unstable conditions. By increasing dynamic postural control and balance, and producing a more coordinated and consistent pattern of movement during movement, the possibility of injury can be prevented (Verhagen, 2005). Gymball exercises can also improve core muscle activation and physical function, because it improves a sense of balance and stimulates the ability of proprioceptors (Behm et al., 2002; Kim et al., 2014). In training, the core muscles are usually not trained or activated during training. In the use of gym ball, activation of the core muscles is maximized, which requires more strength, balance and increased joint stability and can activate muscles according to their functions such as the abdominal muscles and intervertebral muscles.

From the results of data analysis, it is known that the significance $0.000<0.05$, which means that there is a significant effect on balance. The greater instability due to the influence of the gym ball results in the body automatically spurring the neuromuscular system to work bigger, increasing activation of the external obliques, transversus abdominals, internal oblique, erector spinae and rectus abdominals, increasing disturbances to the center of mass of the body. Efforts are required to counter the destabilizing effects of gravity and distraction during exercise movement. Determine the position of the body's center point of gravity relative to the gravitational force and instability device, and then carry out a coordinated 
motion to correct the deviation of the center of gravity in the body. Physiological systems and processes used in visual, vestibular and somatosensory balance, muscle and joint proprioception. in line with the research of (Behm et al., 2002), state that the main purpose of instability training is to increase core stability. The main objective of a training program with the physioball is not necessarily to increase strength but to gain stability, improve balance, and increase proprioceptive abilities.

The main benefit is that when the body is challenged to stabilize the position when doing movements in an unstable position on the gym ball, this system will work harder and more activity in an unstable environment where the stimulation of the stabilizers is reduced, the instability of the ball itself is considered to quickly activate the small muscles. It is not often used and stimulates proprioception and somato sensory systems to maintain balance, proprioceptive systems rely on information from joints and muscles to coordinate subconscious reflexes to maintain balance, contributing to an increase in static and dynamic balance abilities (Behm et al., 2002; Lee, 2008; Sundstrup et al., 2012), Elastic resistance can provide adequate additional weight when making movements on the ball become an effective global muscle strengthening exercise

Results of data analysis of flexibility ability, it is known that the sig value. $0.000<0.05$, which means that there is a significant effect. Gym ball provides a variety of motion combinations that function to expand the range of motion of joints and the level of difficulty of movement. Improvements in flexibility in ability can be associated with dynamic exercises performed. These exercises increases joint stability where gym ball exercises lead to activation of the abdominal and intervertebral muscles and improvement of motor control. Most attempts to increase flexibility are aimed at reducing the resistance that occurs from the connective tissue around the joint. As a muscle has the most elastic tissue, most of the efforts to increase flexibility are aimed at the muscles, the improvement in flexibility in the ability to be associated with dynamic exercises performed on a gymball that provide a wide range of joint ranges of motion as well as levels of movement difficulty.

The greatest enhancement contribution of gym ball exercise improves balance and flexibility. The results of research by (Sekendiz et al., 2007), using gym ball with a training duration of 45 minutes, 3 days / week, for 12 weeks. The results of multivariate analysis revealed significant differences $(\mathrm{p} \leq 0.05)$ abdominal endurance (curl-up test), lower back muscle endurance (modified Sorensen test), lower leg endurance (repetitive squat test), lower back flexibility (sit and reach test), and dynamic balance (functional reach test). (Stathokostas et al., 2012), also revealed that the results of a 12 -week training program significantly improved lower body flexibility, dynamic balance, and strength in adults, static balance significantly increased joint mobility ( $22.5 \%$ in goniometer) and section flexibility. Lower body (25.73\% in the sit and reach test).

The results of the data analysis of strength of the abdominal muscles show the sig value. $0.000<0.05$, shows that there is a significant effect on. Analysis on the use of gym ball has the property of causing greater muscle activation. The advantages of an unstable training environment will be based on neuromuscular adaptations with 
increased strength. Increased strength can be associated with an increase in the cross-sectional area of the muscles to contract with the weight of exercise and improved neuromuscular coordination, an increase in instability at the fulcrum during gym ball training resulting in increased activation of the external obliques, transversus abdominus, internal obliques, erector spinae and rectus abdominus, possibly an associated increase. With neural adaptations, to carry out constant motion requires efforts to counter the destabilizing effects of gravity and disturbances during movement.

The activation rate of these muscles is high in the rectus abdominis (RA), transversus abdominis (TA), lumbar multifidus (MUL), erector spinae (ES), and the internal (IO) or external oblique (EO) during exercise, which is in line with our previous definition. It is a "local stabilizer" that provides stability to the pelvis when performing hip extension movements. Consequently, this exercise is highly recommended for strengthening purposes, given the high activity levels observed not only in the core muscles, but also in the chest and lower limbs. Rectus abdominis activity ranging from $30-60 \%$ has been reported in studies for abdominal exercises over the gymball (Ratamess et al., 2009).

The instability device can place a lower load on the extremities, but results in higher activation of the core muscles. The erector spinae, external oblique, rectus abdominis and quadratus lumborum have long levers and the arm when large is responsible for producing force, producing power over a greater range of motion. Agreeing with the results of the study of (Behm et al., 2011), increased strength can be associated with an increase in the cross-sectional area of muscles in contracting withstand exercise loads and improving neuromuscular coordination. Whereas (Granacher et al., 2013), in his study doing 9 weeks of core strength training on unstable surfaces in adults (age: 63-80 years). The experimental group had significantly increased torsion muscle strength, spinal mobility, functional mobility, and dynamic balance. These exercises induce greater muscle activity than the stability device on the floor, which suggests that adding a ball achieves the instability needed to increase muscle strength (Youdas et al., 2018).

This result of study analysis can be concluded that the gym ball causes instability when a body segment away from the center of the ball is sufficient to increase the prime mover activity associated with muscle activation according to the form of the movement. Muscle recruitment tends to be greater during the eccentric phase compared to the isometric phase; this may explain the increased muscle activity. The lower ball placement not only requires a greater proportion of the hips to be lifted during the crunch movement but also requires greater stabilization of the torso in a horizontal position because there is no support from either the floor or the ball.

Greater degree of muscle stress on the instability device using this gymball, this may form the basis for an enhanced balancing effect after training. Improvements in flexibility can be attributed to dynamic exercises performed on gymballs that provide an increased range of motion. The instability in a gymball leads to muscle activation and improved motor control, which ultimately leads to the use of large muscle strength during training and leads to increased strength and balance abilities as a result of the 
instability of the ball. Gymball exercises that involve isometric actions of muscles, small weights, prolonged tension and repetitions of circuit training alone lead to increased strength in the abdominal muscles and leg muscles.

The instability device using a gym ball with the circuit training method can be used as a reference training program to improve balance, flexibility, abdominal muscle strength, abdominal muscle endurance and leg muscle endurance, additional exercise programs to increase flexibility, increase control-mobility of the torso and pelvic segments. The greatest contribution of gymball exercise allows for improved core stability and muscle balance, strength, flexibility and endurance. This study has shown that no single muscle can be identified as more important for spinal stability during various body movements during exercise. The ability of muscle groups, especially weak core muscles, is believed to interfere with energy transfer, reduce inefficiency in movement during exercise and reduce the increased risk of injury. In training research results it is necessary to evaluate clear evidence for a clear muscle recruitment pattern associated with muscle performance.

\section{Conclusions}

This study concern on the adaptation caused by the instability device program using a gym ball and a stability device using a mattress. This study states that it is widely assumed that the ability of muscle groups, especially weak core muscles, is believed to interfere with energy transfer, reduce inefficiencies in sports movements and contribute to an increased risk of injury.

Muscles with balance, strength, flexibility and joints with greater integrity are less prone to injury, means that the muscles around the joint tend to prioritize stability over power production. There is a significant increase in the instability device using a gym ball compared to the stability using a conventional mat and group, for the following reasons: reduced contact area, increased disturbance of activity due to unstable surfaces, and control of center of gravity at body points with limited support from the ground of gym ball instability. Progressive fatigue throughout training sessions with the ciruit training method affects muscle performance resulting in a greater level of physiological ability to control core muscles.

This research review provides a selection of exercises for muscle activation, especially the larger core muscles based on six different types of exercise (stable device using a mat and instability device using a gym ball) to improve physical condition abilities.

Comment: Regarding the subject of this research, the subject is male students not female, the average weight and height of Indonesian male student is shorter than most of other country.

\section{References}

Behm, D. G., Anderson, K., \& Curnew, R. S. (2002). Muscle Force and Activation Under Stable and Unstable Conditions. Journal of Strength and Conditioning Research, 16(3), 416-422. https://doi.org/10.1519/1533-4287(2002)016<0416:MFAAUS>2.0.CO;2 
Behm, D. G., Drinkwater, E. J., Willardson, J. M., \& Cowley, P. M. (2011). The Role of Instability Rehabilitative Resistance Training for the Core Musculature. Strength \& Conditioning Journal, 33(3), 72-81. https://doi.org/10.1519/SSC.0b013e318213af91

Brentano, M. A., Cadore, E. L., Da Silva, E. M., Ambrosini, A. B., Coertjens, M., Petkowicz, R., Viero, I., \& Kruel, L. F. M. (2008). Physiological Adaptations to Strength and Circuit Training in Postmenopausal Women With Bone Loss. Journal of Strength and Conditioning Research, 22(6), 1816-1825. https://doi.org/10.1519/JSC.0b013e31817ae3f1

Campos de Oliveira, L., Gonçalves de Oliveira, R., \& Pires-Oliveira, D. A. de A. (2015). Effects of Pilates on Muscle Strength, Postural Balance and Quality of Life of Older Adults: a Randomized, Controlled, Clinical Trial. Journal of Physical Therapy Science, 27(3), 871-876. https://doi.org/10.1589/jpts.27.871

Cruz-Ferreira, A., Fernandes, J., Laranjo, L., Bernardo, L. M., \& Silva, A. (2011). A Systematic Review of the Effects of Pilates Method of Exercise in Healthy People. Archives of Physical Medicine and Rehabilitation, 92(12), 2071-2081. https://doi.org/10.1016/j.apmr.2011.06.018

Freeman, J., Gear, M., Pauli, A., Cowan, P., Finnigan, C., Hunter, H., Mobberley, C., Nock, A., Sims, R., \& Thain, J. (2010). The Effect of Core Stability Training on Balance and Mobility in Ambulant Individuals with Multiple Sclerosis: A Multi-Centre Series of Single Case studies. Multiple Sclerosis Journal, 16(11), 1377-1384. https://doi.org/10.1177/1352458510378126

Granacher, U., Gollhofer, A., Hortobágyi, T., Kressig, R. W., \& Muehlbauer, T. (2013). The Importance of Trunk Muscle Strength for Balance, Functional Performance, and Fall Prevention in Seniors: A Systematic Review. Sports Medicine, 43(7), 627-641. https://doi.org/10.1007/s40279-013-0041-1

Herrington, L., \& Davies, R. (2005). The Influence of Pilates Training on The Ability to Contract The Transversus Abdominis Muscle in Asymptomatic Individuals. Journal of Bodywork and Movement Therapies, 9(1), 52-57. https://doi.org/10.1016/j.jbmt.2003.12.005

Hyun, J., Hwangbo, K., \& Lee, C.-W. (2014). The Effects of Pilates Mat Exercise on the Balance Ability of Elderly Females. Journal of Physical Therapy Science, 26(2), 291-293. https://doi.org/10.1589/jpts.26.291

Kim, S. G., Yong, M. S., \& Na, S. S. (2014). The Effect of Trunk Stabilization Exercises with a Swiss Ball on Core Muscle Activation in the Elderly. Journal of Physical Therapy Science, 26(9), 1473-1474. https://doi.org/10.1589/jpts.26.1473

Kloubec, J. A. (2010). Pilates for Improvement of Muscle Endurance, Flexibility, Balance, and Posture. Journal of Strength and Conditioning Research, 24(3), 661-667. https://doi.org/10.1519/JSC.0b013e3181c277a6

Lee, S. (2008). Effect of 12 Week Gymball Exercise Physical Fitness And Balance In Elderly. Kook-Min University.

Phrompaet, S., Paungmali, A., Pirunsan, U., \& Sitilertpisan, P. (2011). Effects of Pilates Training on Lumbo-Pelvic Stability and Flexibility. Asian Journal of Sports Medicine, 2(1). https://doi.org/10.5812/asjsm.34822

Queiroz, B. C., Cagliari, M. F., Amorim, C. F., \& Sacco, I. C. (2010). Muscle Activation During Four Pilates Core Stability Exercises in Quadruped Position. Archives of Physical Medicine and Rehabilitation, 91(1), 86-92. https://doi.org/10.1016/j.apmr.2009.09.016

Ratamess, N., Alvar, B. A., Evetoch, T. K., \& Housh, T. J. (2009). Progression Models in Resistance Training for Healthy Adults. Medicine \& Science in Sports \& Exercise, 41(3), 687-708. https://doi.org/10.1249/MSS.0b013e3181915670

Romero-Arenas, S. (2013). Impact of Resistance Circuit Training on Neuromuscular, Cardiorespiratory and Body Composition Adaptations in the Elderly. Aging and Disease, 04(05), 256-263. https://doi.org/10.14336/AD.2013.0400256

Segal, N. A., Hein, J., \& Basford, J. R. (2004). The Effects of Pilates Training on Flexibility and Body Composition: An Observational study 11 No commercial party having a direct financial interest in the results of the research supporting this article has or will confer a benefit upon the authors(s) 0 . Archives of Physical Medicine and Rehabilitation, 85(12), 1977-1981. https://doi.org/10.1016/j.apmr.2004.01.036

Sekendiz, B., Altun, Ö., Korkusuz, F., \& Akın, S. (2007). Effects of Pilates Exercise on Trunk Strength, Endurance and Flexibility in Sedentary Adult Females. Journal of Bodywork and Movement Therapies, 11(4), 318-326. https://doi.org/10.1016/j.jbmt.2006.12.002 
Sherrington, C., Whitney, J. C., Lord, S. R., Herbert, R. D., Cumming, R. G., \& Close, J. C. T. (2008). Effective Exercise for the Prevention of Falls: A Systematic Review and Meta-Analysis. Journal of the American Geriatrics Society, 56(12), 2234-2243. https://doi.org/10.1111/j.15325415.2008.02014.x

Stathokostas, L., Little, R. M. D., Vandervoort, A. A., \& Paterson, D. H. (2012). Flexibility training and functional ability in older adults: A systematic review. Journal of Aging Research, 8(1), 1-30. https://doi.org/10.1155/2012/306818

Sundstrup, E., Jakobsen, M. D., Andersen, C. H., Jay, K., \& Andersen, L. L. (2012). Swiss Ball Abdominal Crunch with Added Elastic Resistance is an Effective Alternative to Training Machines. International Journal of Sports Physical Therapy, 7(4), 372-380.

Verhagen, E. A. L. M. (2005). An Economic Evaluation of a Proprioceptive Balance Board Training Programme for The Prevention of Ankle Sprains in Volleyball. British Journal of Sports Medicine, 39(2), 111-115. https://doi.org/10.1136/bjsm.2003.011031

Youdas, J. W., Coleman, K. C., Holstad, E. E., Long, S. D., Veldkamp, N. L., \& Hollman, J. H. (2018). Magnitudes of Muscle Activation of Spine Stabilizers in Healthy Adults During Prone on Elbow Planking Exercises with and Without a Fitness Ball. Physiotherapy Theory and Practice, 34(3), 212222. https://doi.org/10.1080/09593985.2017.1377792 